\title{
CONTRIBUIÇÃO DO BIM PARA A SUSTENTABILIDADE ECONÔMICA DE EDIFICAÇÕES
}

THE CONTRIBUTION OF BIM TO ECONOMIC SUSTAINABILITY OFBUILDINGS

\section{Leticia Mattana, Esp. (UFSC); Lisiane Ilha Librelotto, Dra. (UFSC)}

\author{
Palavras Chave \\ Sustentabilidade Econômica; BIM; Construção
}

\section{Key Words}

Economic Sustainability; BIM; Construction

\section{RESUMO}

A sustentabilidade pode ser avaliada em diferentes dimensões, variando em quantidade de desdobramentos conforme o autor, mas no mínimo é caracterizada como social, ambiental e econômica. Apesar da importância do tema sustentabilidade, muitas empresas de construção ainda não adotaram tais práticas pela busca de soluções para os problemas gerados por suas atividades. A tecnologia BIM (Building Information Modelling) desponta no setor como uma importante ferramenta integrando o ciclo de vida de edificações e a avaliação da sustentabilidade. O objetivo deste artigo é determinar a contribuição da tecnologia BIM para avaliação da sustentabilidade econômica de edificações. A metodologia MASP-HIS de autoria da pesquisadora Carvalho (2009) foi utilizada para determinar os parâmetros considerados na sustentabilidade econômica das edificações. Pretende-se levantar e apresentar bibliografias, através de um processo de revisão sistemática, que comprovem a contribuição das ferramentas BIM para a sustentabilidade econômica, conforme definições propostas pela metodologia MASP-HIS. Este conhecimento é importante porque aborda o uso do BIM para obter edificações mais sustentáveis, e busca atender expectativas atuais do mercado da construção.

\section{ABSTRACT}

Sustainability in construction can be evaluated in different aspects, varying according to the authors. Some usual aspects, for example, are the social, environmental and economic ones. Despite the importance of sustainability, many construction companies have not adopted practices to solve the problems generated by their activities. BIM technology (Building Information Modelling) emerges in the sector as an important tool integrating the life cycle of a buildings and the evaluation of sustainability. The objective of this paper is to clarify the contribution of BIM technology to evaluate the economic sustainability of buildings. The MASP-HIS methodology authored by Carvalho (2009) will be used to determine which parameters should be evaluated in this study. It is intended to present bibliographies that allow evaluating the economic sustainability of an enterprise using BIM tools, according to definitions of MASP-HIS methodology. This knowledge is important because addresses the use of BIM to obtain more sustainable buildings, as well as to meet the expectation of the construction market. 


\section{INTRODUÇÃO}

Os problemas ambientais associados aos fenômenos climáticos intensos trouxeram consigo o clamor por construções mais sustentáveis, que causem menor impacto ao meio ambiente e que contribuam para um futuro mais equilibrado para o planeta. $O$ setor da construção civil, tanto brasileiro como mundial, busca encarar este desafio através da minimização dos impactos ambientais, do uso racional dos recursos naturais, da redução de entulho e das emissões de $\mathrm{CO} 2$, do incentivo aos programas educacionais para a mão de obra, entre outros. (MARCOS, 2015; VIANA, 2013)

Apesar da importância do tema sustentabilidade, muitas empresas de construção ainda não adotaram tais práticas pela busca de soluções para os problemas sociais, ambientais e econômicos gerados por suas atividades. Alguns motivos que justificam este fato são a falta de dados e de quantificação dos benefícios da atividade, e também a resistência cultural do setor às mudanças. Este conservadorismo do setor, apontado por alguns autores, dificulta a inserção de inovações tecnológicas, o que mantém o setor em atraso em relação aos demais setores econômicos do mercado. (MARCOS, 2015; VIANA, 2013)

Avaliar a sustentabilidade do edifício implica na análise de muitas variáveis que requerem tempo do processo de desenvolvimento do produto e atuação de múltiplos profissionais. Com o uso do processo BIM, "Building Information Modelling", ou em português "Modelagem da Informação da Construção", de acordo com Eastman et al (2014) é possível obter uma avaliação consistente, fidedigna e que permite a decisão em tempo hábil, preferencialmente na etapa de projeto.

Deve-se considerar que, por ser uma tecnologia inovadora, a Modelagem BIM aplicada na prática ainda é incipiente. Existe uma dificuldade de trabalho de forma colaborativa, que em parte deve-se a cultura projetual estabelecida no setor. Somam-se a isto, as restrições, possibilidades de integração via plataforma IFC e particularidades de cada softwares. (OLIVEIRA; SCHEER; TAVARES, 2015)

BIM é uma inovação no setor da engenharia, arquitetura e construção. Com o BIM todo o ciclo de vida dos empreendimentos passará por mudanças em seus processos. Essas mudanças nos processos incluem etapas desde a concepção, a elaboração e o detalhamento dos projetos das diversas disciplinas envolvidas (arquitetônicos e complementares), o planejamento e o orçamento da obra, a execução da obra e a ocupação, considerando a manutenção, a operação e, por fim a demolição desta edificação. (MANZIONE, 2013)
Existem diversos softwares ou plataformas tecnológicas que auxiliam no desenvolvimento do processo BIM e que permitirão a criação de um modelo 3D paramétrico. Este modelo, representado em três dimensões, é uma construção virtual do empreendimento e contém diversas informações incorporadas a ele, e será aplicado às diferentes disciplinas envolvidas no processo de projeto. (KASSEM; AMORIM, 2015)

Como exemplo de informações dos modelos, podem-se citar os materiais que serão utilizados na obra, a extração das quantidades de cada um destes materiais, a compatibilização de projetos (hidráulico, elétrico, arquitetônico, estrutural...) e a solução de conflitos existentes neste modelo unificado, entre outras. (EASTMAN et al., 2014)

Com o modelo BIM unificado surgem alguns benefícios. Um deles é a integração dos diversos profissionais em trabalhos colaborativos, já que o mesmo modelo será utilizado por todos os profissionais envolvidos no processo. A modelagem BIM mostra-se como um processo que auxiliará nos trabalhos relativos às edificações e promete trazer mudanças significativas ao processo e aos profissionais envolvidos. (MANZIONE, 2013)

Tendo em vista as preocupações com o desenvolvimento de construções sustentáveis e que busquem minimizar os impactos causados pelas atividades da construção civil, o uso de tecnologia BIM ganha força como uma possível solução para a questão. Além disso, esta inovação na construção amplia a perspectiva de um futuro meIhor e mais equilibrado para o planeta. Para Marcos (2015) é através desta nova tecnologia que será possível obter a racionalização dos processos e atender às expectativas do mercado da construção.

\section{REVISÃO BIBLIOGRÁFICA}

\subsection{A sustentabilidade e suas dimensões}

A sustentabilidade pode ser definida pela busca por um meio ambiente mais equilibrado e justo para o planeta, e caracteriza-se em diferentes dimensões ou aspectos, que quando somados resultam no desenvolvimento sustentável. O Relatório de Brundtland, publicado em 1987, descreve que o desenvolvimento sustentável é aquele que garante que atenderá "as necessidades do presente sem comprometer a capacidade de as gerações futuras atenderem também às suas...". (BRUNDTLAND et al., 1987)

Carvalho (2009) e Sachs (1993) definem desenvolvimento sustentável como aquele que se preocupa com o uso racional dos recursos naturais e que abrange a 
questão sociocultural, política, estética e outras. Esses autores mostram que a integração e dependência entre as dimensões da sustentabilidade é que originam a construção sustentável, conforme apresentado na figura 1, na qual se divide a sustentabilidade em 3 grupos: econômica, ambiental e sociocultural.

Librelotto (2005) também considera três principais dimensões da sustentabilidade: a social, a econômica e a ambiental. A autora define a dimensão social como a que envolve responsabilidade social e gestão das pessoas, a dimensão ambiental como a que se preocupa com a preservação dos ecossistemas e a redução de impactos ao meio ambiente, e a dimensão econômica que garante o retorno financeiro dos investimentos aos proprietários, comunidade e outros envolvidos no processo.

Carvalho (2009) menciona que a maneira como se constrói, se projeta e se opera uma edificação influencia no uso dos recursos naturais e na saúde da população, e por isso a autora justifica a importância de obter um desenvolvimento sustentável para o setor da construção. Para John (2006) apud Carvalho (2009) a inovação tecnológica e o uso de novas tecnologias, tanto em sala de aula como na vida profissional, deve reinventar a sustentabilidade e facilitar a busca pelas certificações dos produtos.

\section{CONSTRUÇÃO SUSTENTÁVEL}

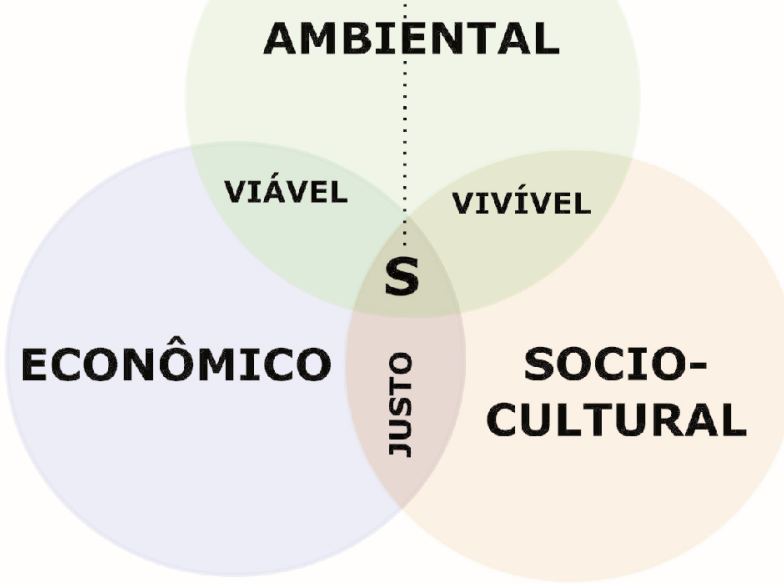

Fonte: Adaptado de Carvalho (2009), Sacks (1993) e Librelotto (2005)

\subsection{A Sustentabilidade Econômica}

Librelotto (2005) define dimensão econômica como aquela que "associa a estrutura-conduta-desempenho a

garantia de retorno dos investimentos aos intervenientes do processo (proprietários, clientes, funcionários e comunidade em geral)".

Já os indicadores de sustentabilidade do IBGE consideram a dimensão econômica da sustentabilidade nos aspectos relacionados ao uso de recursos naturais, à questão da geração de resíduos, ao uso de energia e ao desempenho econômico do país. Esta dimensão avalia a eficiência dos processos produtivos e o consumo de recursos. (IBGE, 2015)

Em relação à dimensão econômica da sustentabilidade, Carvalho (2009) desenvolveu uma metodologia para avaliação da sustentabilidade de habitações de interesse social e aborda aspectos econômicos nesta metodologia, que foi chamada de MASP-HIS. Ela quantifica a sustentabilidade econômica na metodologia da pesquisa e considera como fatores para avaliação sustentável do pilar econômico o fortalecimento da economia, a viabilidade econômica, o custo de construção/manutenção/operação e os critérios econômicos para empresas de projetos, conforme apresentado na Figura 2.

\section{Figura 2: Aspectos econômicos da sustentabilidade. ASPECTOS ECONÔMICOS}

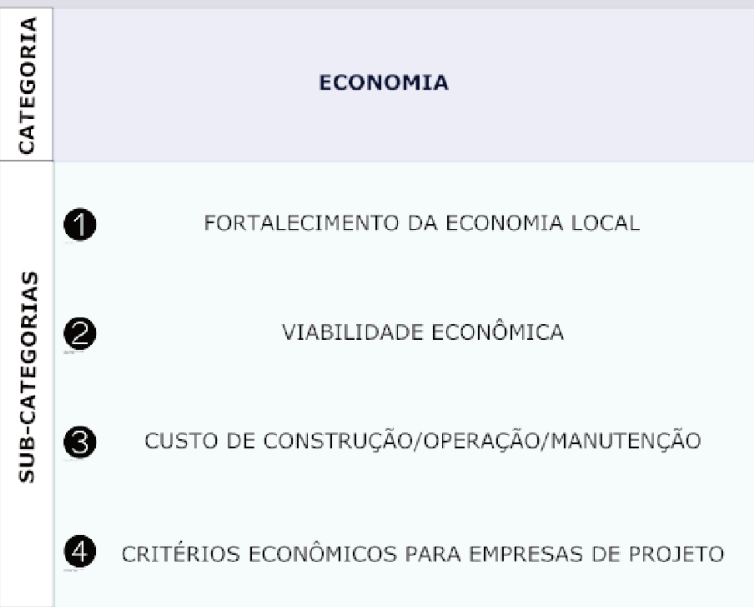

Fonte: Adaptado de Carvalho (2009)

Em relação ao fortalecimento da economia local, a autora considera positivo o uso de materiais locais, a existência de infraestrutura básica no local e a geração de emprego e renda. Para a viabilidade econômica, a autora considera favorável o custo acessível com a realidade local, a existência de retorno do investimento, a inexistência de custos com implantação de infraestrutura, a consideração no orçamento de ampliações nas infraestruturas existentes e a existência de subsídios fiscais para os que adotarem medidas sustentáveis. (CARVALHO, 2009) 
Sobre o custo de construção, operação e manutenção, Carvalho (2009) expõe que a existência de soluções que diminuam o custo de construção/manutenção/operação e a economia de água e energia são pontos positivos para a sustentabilidade econômica. Em relação aos critérios econômicos para empresa de projeto, a autora considera favorável a existência de ações sustentáveis internas à empresa, tais como a economia de água e energia, a reciclagem, políticas de critérios sustentáveis nas compras e verbas destinadas à melhoria do desempenho sustentável da empresa e dos trabalhadores (CARVALHO, 2009). Todos os aspectos abordados por Carvalho contribuem para a avaliação da sustentabilidade econômica e ajudam a quantificá-la e mensurá-la em uma edificação.

\subsection{O desenvolvimento de projetos sustentáveis}

Melhado, Uechi e de Paula (2013) mencionam que a cada dia crescem as demandas em termos de melhorar o desempenho das edificações no setor da construção brasileira. Pode-se citar a demanda pelo cumprimento da Norma de Desempenho, por exemplo, e a crescente busca por edificações mais sustentáveis, principalmente em seu aspecto ambiental e através dos selos de certificações ambientais.

A NBR 15575 considera a sustentabilidade como durabilidade, manutenibilidade e impacto ambiental, excluindo de seu conceito os aspectos relativos à habitabilidade, conforto, funcionalidade e flexibilidade, restringindo o conceito, na contramão de outras normas internacionais como as normas ISO da série 9000, 14000 e 26000, já traduzidas para o Brasil. Por estas normas, sustentabilidade em sua dimensão econômica é representada pela Gestão da Qualidade, na dimensão Social pelas normas de Gestão de Riscos, Saúde e Segurança Ocupacional e pela Responsabilidade Social Empresarial. Na dimensão ambiental, associam-se as normas de Gestão Ambiental da série ISO 14000. (ABNT, 2013)

A preocupação com a sustentabilidade tem crescido na indústria da construção, baseada nos conceitos de projetos que consideram fatores sociais, econômicos e ambientais nas suas estratégias. O impacto dos custos resultantes de projetos de edificações sustentáveis é um dos recursos que os projetistas deveriam considerar quando estão projetando um novo empreendimento. (ALSAYYAR; JRADE, 2015)

Librelotto (2005) enfatiza que para obter vantagem competitiva sustentável, não basta pensar apenas na dimensão econômica, mas sim deve-se considerar também as questões sociais e ambientais, e o equilíbrio delas como um todo.

Azevedo (2009) comenta a questão, sugerindo que a sustentabilidade é considerada uma demanda para os projetos e obras. Com esta nova demanda sustentável para os projetos, estes passam a atender outros requisitos que se adicionam às necessidades prévias do cliente, para além do usual, e podem ser vistos como oportunidades de inovação no processo do setor da construção.

Quando as análises são realizadas nos estágios iniciais do ciclo de vida do empreendimento, permite-se maior controle sobre o impacto no custo. Isso ocorre através das análises de múltiplos critérios de projetos, como os materiais usados, as tecnologias escolhidas e a definição dos espaços, que afetam diretamente os custos do empreendimento. (ALSAYYAR; JRADE, 2015)

Melhado, Uechi e de Paula (2013) também descrevem que o uso de BIM no processo de projeto pode ser considerado uma demanda de mercado e uma possível solução para cumprir com as novas exigências.

Para Carvalho (2009) a questão do desenvolvimento sustentável deve ser vista nas fases de planejamento, na qual é possível analisar a participação do usuário nas decisões, nas características do local, na disposição de recursos financeiros e ambientais, além de analisar os impactos da ocupação futura no local e a existência de infraestrutura e outros aspectos relevantes.

Na fase de projeto, Carvalho (2009) menciona que deve-se escolher o processo construtivo que gera menores impactos, e que este é o momento decisivo para buscar os princípios da sustentabilidade para a futura edificação. Além disso, ela complementa dizendo que o processo de projeto é complexo e possui deficiências, que devem ser sanadas através da busca por maior integração entre as disciplinas envolvidas, entre as equipes de profissionais, e a busca pela melhoria na gestão como um todo.

Em seu modelo de avaliação da sustentabilidade para projetos de habitação de interesse social, Carvalho propõe uma série de indicadores que vão desde o consumo de recursos e o fluxo de massa, a qualidade interna da habitação (conforto e saúde), a qualidade do produto habitação, aspectos sociais e culturais, geração de renda e responsabilidade social, aspectos políticos institucionais e segurança, custos de construção, operação e manutenção da construção. Esta abordagem, portanto, pode ser considerada bem mais ampla do que a visão dada pela Norma de Desempenho.

Ao se considerar o âmbito da avaliação da sustentabilidade, por exemplo, principalmente na aplicação de 
metodologias mais amplas, como a de Carvalho (2009) ou mesmo a de Librelotto, Sanon e Ferroli (2017), dada pelo Modelo ESA Edifícios, o emprego de BIM pode viabilizar a adoção do método em maior escala. Tal fato deve-se a complexidade de medição em ambos os modelos, com o uso do BIM agindo como um agente facilitador.

Para Azevedo (2009) uma grande contribuição para atingir as novas demandas de mercado é o uso de BIM em todo o ciclo de vida de um empreendimento. Assim, é possível "controlar melhor os prazos de execução e os custos", bem como questões relacionadas à sustentabilidade.

Entretanto, o emprego do BIM na avaliação da sustentabilidade das edificações restringe-se, em uma primeira análise a obtenção de dados confiáveis. Assim, ao desmembrar-se a sustentabilidade em sua dimensão mais conhecida (ou historicamente precursora), a dimensão econômica, deveria ser possível à extração de quantitativos precisos, independente do software utilizado, de forma a permitir a análise dos custos e talvez, sua extrapolação para avaliação do impacto ambiental a partir da associação destas quantidades com dados de inventários de materiais.

\subsection{A Modelagem da Informação da Construção}

A Modelagem da Informação da Construção ou Building Information Modeling - BIM - é uma inovação no setor da engenharia, arquitetura e construção, que promete trazer mudanças nos processos de gestão de obras e projetos, englobando todo o ciclo de vida dos empreendimentos.

Essas mudanças nos processos que incluem etapas do ciclo de vida da edificação contemplam desde a concepção, a elaboração e o detalhamento dos projetos das diversas disciplinas envolvidas, como por exemplo, o arquitetônico e os complementares, o planejamento e o orçamento da obra, a execução da obra e a ocupação, considerando a manutenção, a operação e, por fim a demolição desta edificação, conforme figura 3.

Santos, Antunes e Balbinot (2014) referem-se ao BIM como "um banco de dados unificado, onde quaisquer informações relacionadas ao edifício podem ser criadas ou extraídas".

\section{Ciclo de Vida - BIM}

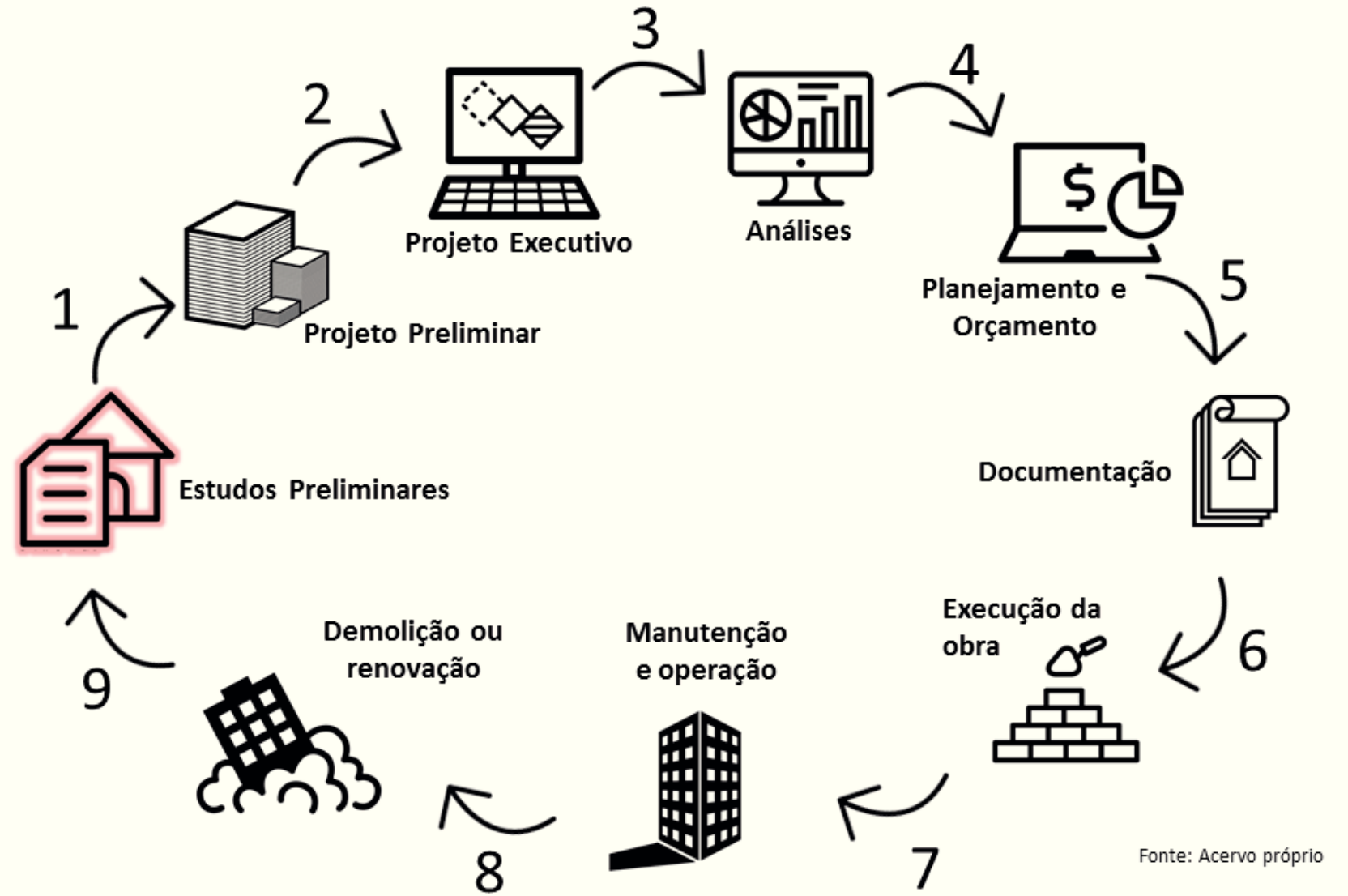

Fonte: Adaptado de Rozenfeld e outros (2006), Librelotto (2009) e The Noum Project (2017) 
Ao considerar o modelo BIM como um banco de dados, pode-se dizer que em consequência disto este modelo necessita de gestão para estas informações, e, portanto, BIM é também um sistema de gestão das várias informações que estão integradas no modelo. (WONG; FAN, 2013)

Para Wong e Fan (2013), apud NBIMS (2016), BIM pode ser considerado um produto porque é um modelo completo que descreve uma edificação e será entregue para um cliente. Outra definição proposta por eles é a de que BIM é um processo de projeto, visto que considera as diversas atividades que são realizadas e as equipes colaborativas envolvidas até que o modelo esteja completo para ser entregue.

No livro Manual de BIM, há uma definição para processo BIM: "Processo que se apoia nas informações geradas por uma ferramenta de projeto BIM para análise, detalhamento para fabricação, estimativa de custos, cronograma, entre outros". (EASTMAN et al., 2014)

Para Rozenfeld e outros (2006), o termo processo caracteriza "um conjunto de atividades realizadas em uma sequência lógica com o objetivo de produzir um bem ou serviço que tem valor para um grupo específico de clientes".

No mesmo Manual de BIM, há uma definição interessante para o entendimento de BIM como uma ferramenta. Os autores definem que "ferramenta BIM" é um termo usado quando se faz referência aos aplicativos de manipulação dos modelos de construção, ou seja, quando se refere aos softwares BIM. A ideia recebe a complementação de que o termo pode ser ajustado para "ferramenta de projeto BIM", indicando novamente os softwares BIM usados para fins projetuais. (EASTMAN et al., 2014)

Rozenfeld e outros (2006) também definem o termo ferramenta, que para eles é um meio para descrever os sistemas de informações, ou seja, as ferramentas de informática, que por sua vez podem aumentar a eficácia do processo de desenvolvimento de um determinado produto.

Com base nesta definição, não é interessante rotular BIM apenas como uma ferramenta, pois esta definição refere-se somente ao software de trabalho, enquanto que o conceito de BIM é mais amplo do que isso e considera o processo de trabalho como um todo.

Existem várias definições sobre BIM, dos quais se destacam os sugeridos por Eastman e outros (2014, pg.13), que definem como BIM "uma tecnologia de modelagem e um conjunto associado de processos para produzir, comunicar e analisar modelos de construção", e por AIA - The American Institute of Architects (2008), que considera BIM como uma representação digital das características físicas e funcionais de um projeto. O documento divulgado por AIA complementa que Building Information Modeling refere-se ao processo e às tecnologias usadas para criar um modelo.

Existem diversos softwares ou plataformas tecnológicas que auxiliam no desenvolvimento do processo BIM e que permitirão a criação de um modelo 3D paramétrico. Este modelo, representado em três dimensões, é uma construção virtual do empreendimento e contém diversas informações incorporadas a ele. Este modelo paramétrico poderá ser aplicado às diferentes disciplinas envolvidas no processo de projeto. (KASSEM; AMORIM, 2015)

Como exemplo de informações que existirão nestes modelos, pode-se citar a definição dos materiais que serão utilizados na obra, a extração das quantidades de cada um destes materiais, a compatibilização de projetos (hidráulico, elétrico, arquitetônico, estrutural...) e a solução de conflitos existentes neste modelo, entre outras.

Com um modelo unificado 3D surgem alguns benefícios devido ao uso de BIM. Um deles é a integração dos diversos profissionais em trabalhos colaborativos, já que o mesmo modelo será utilizado por todos os profissionais envolvidos no processo. Além disso, a adoção do BIM deve trazer benefícios para a elaboração de edificações mais sustentáveis, através da análise prévia e de questionamentos quanto aos materiais escolhidos e quanto ao uso de energia, por exemplo, além de ser um exercício social, com foco na colaboração entre as pessoas.

\section{PROCEDIMENTO METODOLÓGICO}

Inicialmente, foi realizada uma estruturação dos conceitos envolvidos na aplicação do BIM para extração de informações que permitam avaliar a sustentabilidade de uma edificação. A partir destes conceitos, foram pesquisados trabalhos relacionados à Modelagem da Informação da Construção, que compõem a revisão bibliográfica da pesquisa.

Esta pesquisa ocorreu na forma de revisão sistemática da bibliografia, com o objetivo de identificar o maior número possível de estudos relacionados ao tema. Com isso, foi possível compreender o estado da arte da adoção do BIM e sua contribuição para as construções sustentáveis. Com base no material coletado, redigiu-se este artigo.

A busca foi realizada preliminarmente no Banco de Teses e Dissertações da Capes, utilizando como palavras-chaves a sigla BIM e quantitativos. Nesta primeira associação encontrou-se 10321 resultados. Destes, filtrando-se por área de conhecimento (engenharias, arquitetura, tecnologias e áreas correlatas) para trabalhos publicados 
a partir de 2010, com a inserção da palavra orçamento na busca, obteve-se 2392 resultados. Os principais resultados estão apresentados no Quadro 1.
Foi realizada nova busca no Banco de Teses e Dissertações da Capes, utilizando outras combinações de palavras como a sigla BIM e a palavra DProfiler, que é um

Quadro 1 - Revisão Sistemática no banco de teses e dissertações da CAPES

\begin{tabular}{|c|c|}
\hline \multicolumn{2}{|c|}{ Trabalhos anteriores à Plataforma Sucupira } \\
\hline AUTOR; TÍTULO; ANO & AUTOR; TÍTULO; ANO \\
\hline $\begin{array}{l}\text { WITICOVSKI, Lilian Cristine. Levantamento de quan- } \\
\text { titativos em projeto: uma análise comparativa do } \\
\text { fluxo de informações entre as representações em } \\
\text { 2D e o modelo de informações da construção (BIM). } \\
\text { 2011 }\end{array}$ & $\begin{array}{l}\text { MARTINEZ, Laura Dominguez. BIM aplicado ao } \\
\text { processo de projeto sustentável: um estudo do } \\
\text { segmento de projetos unifamiliares residenciais em } \\
\text { Niterói-RJ. } 2010\end{array}$ \\
\hline $\begin{array}{l}\text { ANDRADE, Ludmila Santos de. A Contribuição dos } \\
\text { sistemas BIM para o planejamento orçamentário } \\
\text { das obras públicas: Estudo de Caso do auditório e } \\
\text { da biblioteca de Planaltina. } 2012\end{array}$ & \\
\hline \multicolumn{2}{|c|}{ Trabalhos posteriores à Plataforma Sucupira } \\
\hline AUTOR; TÍTULO; ANO & CONTEÚDO \\
\hline $\begin{array}{l}\text { DINIZ, Maria da Conceição Mendes. Modelagem da } \\
\text { informação da construção Estudo de Caso - Inspe- } \\
\text { toria da Receita Federal em Jaguarão - RS. } 2013\end{array}$ & $\begin{array}{l}\text { Consiste na análise comparativa entre os métodos de } \\
\text { elaboração de estimativas de custo de obra por m2 e } \\
\text { por sistema BIM. }\end{array}$ \\
\hline $\begin{array}{l}\text { SAKAMORI, Marcelo Mino. Modelagem 5D (BIM) } \\
\text { - Processo de orçamentação com estudo sobre } \\
\text { controle de custos e valor agregado para empreen- } \\
\text { dimentos de construção civil'. } 2015\end{array}$ & $\begin{array}{l}\text { Visa ilustrar o conjunto de processos utilizados para a } \\
\text { modelagem 5D, demonstrando desde a fase de mod- } \\
\text { elagem, passando pela extração de dados do modelo, a } \\
\text { indexação dos dados quantitativos aos custos forne- } \\
\text { cidos pelo banco de dados da SINAPI até a inclusão } \\
\text { de estudos adicionais que servem como uma linha de } \\
\text { base para o controle dos custos do empreendimento }\end{array}$ \\
\hline $\begin{array}{l}\text { COSTA, José Martins Cavalcanti da. Diagnóstico da } \\
\text { Implantação do BIM em Empresas Construtoras com } \\
\text { foco nos Processos de Orçamento, Planejamento e } \\
\text { Controle de Obras. } 2015\end{array}$ & $\begin{array}{l}\text { Este trabalho tem como propósito analisar os princi- } \\
\text { pais impactos da tecnologia BIM sobre os processos de } \\
\text { planejamento, orçamento e controle, bem como apre- } \\
\text { sentar uma representação esquemática do impacto do } \\
\text { BIM sobre o fluxo dos processos estudados. }\end{array}$ \\
\hline $\begin{array}{l}\text { BAIA, Denize Valéria Santos. Uso de ferramentas BIM } \\
\text { para o planejamento de obras da construção civil. } \\
2015\end{array}$ & $\begin{array}{l}\text { Análise das contribuições da utilização de sistemas BIM, } \\
\text { mais precisamente das ferramentas Revit e Navisworks, } \\
\text { além do uso do software MSProject, voltados para o } \\
\text { melhor planejamento de obras da construção civil. }\end{array}$ \\
\hline $\begin{array}{l}\text { PAPADOPOULOS, Nicolas Alexandros. Avaliação da } \\
\text { Metodologia BIM através da Modelagem Paramétri- } \\
\text { ca 3D de um Projeto Convencional. } 2014\end{array}$ & $\begin{array}{l}\text { Ao término desse estudo são obtidos exemplos reais } \\
\text { dos benefícios da modelagem 3D parametrizada. Uma } \\
\text { série de deficiências são detectadas nos documentos } \\
\text { 2D originais. }\end{array}$ \\
\hline $\begin{array}{l}\text { MARCOS, MICHELINE HELEN COT. Metódo de ob- } \\
\text { tenção de dados de impactos ambientais, durante } \\
\text { o processo de desenvolvimento do projeto, através } \\
\text { do uso de ferrramenta BIM. } 2015\end{array}$ & $\begin{array}{l}\text { Desenvolve um método de obtenção de dados de im- } \\
\text { pactos ambientais, durante o processo de desenvolvi- } \\
\text { mento do projeto, através do uso de uma ferramenta } \\
\text { de Modelagem da Informação da Construção ("Building } \\
\text { Information Modeling" - BIM), para auxiliar na tomada } \\
\text { de decisões quanto ao sistema construtivo que propor- } \\
\text { cione menor impacto ambiental. }\end{array}$ \\
\hline
\end{tabular}




\begin{tabular}{|c|c|}
\hline \multicolumn{2}{|c|}{ Trabalhos posteriores à Plataforma Sucupira } \\
\hline AUTOR; TÍTULO; ANO & CONTEÚDO \\
\hline $\begin{array}{l}\text { PEREIRA, Sílvia Maria Soares de Araújo. A contribuição } \\
\text { do BIM no processo de projeto de arquitetura - uma } \\
\text { aplicação ao projeto de biotérios. } 2014\end{array}$ & $\begin{array}{l}\text { Estudou-se o potencial do BIM no processo de projeto, } \\
\text { através da automação de parte dele e da verificação } \\
\text { automática de requisitos do projeto de arquitetura de } \\
\text { biotérios. }\end{array}$ \\
\hline $\begin{array}{l}\text { BIOTTO, Clarissa Notariano. Método para projeto e } \\
\text { planejamento de sistemas de produção na con- } \\
\text { strução civil com uso da modelagem BIM 4D }\end{array}$ & $\begin{array}{l}\text { Desenvolvimento de um método para o projeto e } \\
\text { planejamento de sistemas de produção em empreendi- } \\
\text { mentos de construção, com uso da modelagem BIM 4D. }\end{array}$ \\
\hline $\begin{array}{l}\text { IBARRA, José Fernando Villamayor. Integração de } \\
\text { modelos de processo e produto na fase de con- } \\
\text { strução para o controle da produção com o uso de } \\
\text { BIM }\end{array}$ & $\begin{array}{l}\text { A contribuição deste trabalho está relacionada à } \\
\text { integração entre os resultados dos controles proativos } \\
\text { no canteiro e o modelo BIM do empreendimento, de } \\
\text { forma efetiva e estável. }\end{array}$ \\
\hline $\begin{array}{l}\text { MORAIS, Marcelo de. Método para implementação } \\
\text { de BIM e custeio-meta em habitação de interesse } \\
\text { social. }\end{array}$ & $\begin{array}{l}\text { Base inicial de projeto (conjunto integrado de modelos } \\
\text { BIM) que parte de templates de projeto (que incluem } \\
\text { famílias de componentes e tabelas quantitativas). A } \\
\text { base de projeto é associada à planilhas de gerencia- } \\
\text { mento de valor. }\end{array}$ \\
\hline $\begin{array}{l}\text { MACIEL, Marcelo Augusto Costa. Dificuldades para a } \\
\text { implantação de softwares integradores de projeto } \\
\text { (BIM) por usuários da cidade de Aracaju/Sergipe }\end{array}$ & $\begin{array}{l}\text { O objetivo deste trabalho é analisar quais são as } \\
\text { maiores dificuldades para se implantar a tecnologia } \\
\text { BIM nos escritórios de arquitetura e urbanismo, nos } \\
\text { escritórios que desenvolvem projetos complementares } \\
\text { e nas empresas construtoras, em cidades do Nordeste } \\
\text { do Brasil. }\end{array}$ \\
\hline $\begin{array}{l}\text { MEDEIROS, Sanderson Carvalho Souza de. Integração } \\
\text { de projetos no ensino através do BIM: uma abor- } \\
\text { dagem dos cursos de arquitetura e urbanismo da } \\
\text { UFRN e da UFPB. } 2015\end{array}$ & $\begin{array}{l}\text { Analisar o uso de ferramentas BIM como método de } \\
\text { integração interdisciplinar e o ensino de projeto no } \\
\text { curso de arquitetura. }\end{array}$ \\
\hline $\begin{array}{l}\text { GARCIA, DANILO DOS SANTOS. Arquitetura Performa- } \\
\text { tiva: A Utilização do DProfiler para Elaboração da } \\
\text { Forma Arquitetônica. } 2014\end{array}$ & $\begin{array}{l}\text { Este trabalho faz a abordagem de projeto digital } \\
\text { focando no desempenho de custos, devido à suma } \\
\text { importância do fator orçamentário preliminar para a } \\
\text { execução do edifício e para o lançamento do partido } \\
\text { arquitetônico. }\end{array}$ \\
\hline $\begin{array}{l}\text { BARISON, MARIA BERNARDETE. Introdução de mod- } \\
\text { elagem da informação da construção (BIM) no } \\
\text { currículo: uma contribuição para a formação do } \\
\text { projetista. } 2015\end{array}$ & $\begin{array}{l}\text { Introdução do BIM nos currículos dos cursos de ar- } \\
\text { quitetura e engenharia civil, nas diferentes disciplinas } \\
\text { curriculares. Definição de especialidades BIM. }\end{array}$ \\
\hline
\end{tabular}

software BIM para análise de viabilidade. Nesta associação encontrou-se 1685 resultados. Destes, mantendo-se os mesmos filtros anteriores, obteve-se 192 resultados, dos quais os mais importantes estão apresentados também no Quadro 1. Outras bases foram pesquisadas, tais como Scopus e Springer, que trouxeram novos resultados, com base nas palavras-chaves já citadas e com inserção de novas palavras na busca, como educação, processo de gestão integrada, sustentabilidade entre outros. Os resultados estão no Quadro 1.

\section{RESULTADOS E DISCUSSÕES}

Segundo Carvalho (2009), existem quatro subcategorias que caracterizam a avaliação econômica da sustentabilidade para uma edificação. Estas subcategorias são: fortalecimento da economia local, a viabilidade econômica, o custo de construção, de operação e de manutenção de uma determinada edificação e os critérios econômicos para empresa de projeto.

Para cada subcategoria, realizou-se a busca para identificar a existência de experiências e estudos relativos ao 
BIM que possam contribuir com o resultado almejado da sustentabilidade econômica de um empreendimento. Foram encontrados alguns trabalhos que relatam o uso de ferramentas BIM para tal finalidade. Algumas destas ferramentas estão apresentadas na figura 4 e abrangem todo o ciclo de vida das edificações.

Figura 4 - Variedade de softwares BIM que contribuem com a sustentabilidade econômica de edificações.

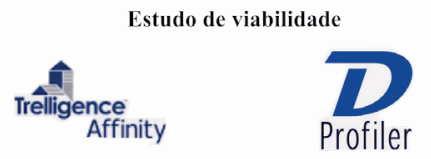

Levantamento de quantidades
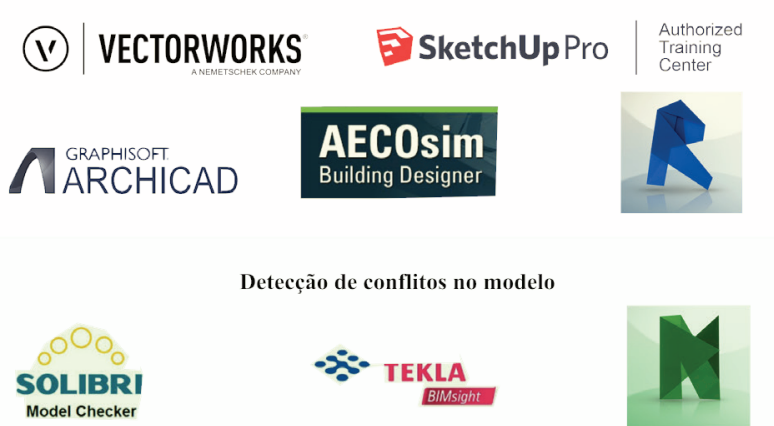

Detecção de conflitos no modelo
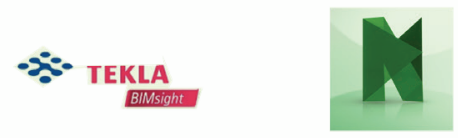

\section{Allplan

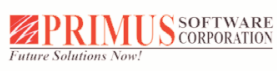

Manutenção e operação da obra

Allplan

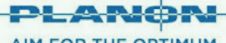
AIM FOR THE OPTIMUM

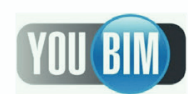

Fonte: Autoras

Esta contribuição do BIM para a sustentabilidade econômica inicia com a viabilidade econômica, através do uso de softwares como o DProfiler e o Trelligence Affinity que trazem o custo estimado de uma obra em etapas prévias do projeto, permitindo analisar a viabilidade para executar determinado empreendimento.

Verificou-se que a adoção do BIM traz grande contribuição em relação aos custos de construção de uma edificação. Uma delas é através do uso de ferramentas de modelagem 3D, nas quais pode-se extrair quantidades diretamente dos modelos. Isso pode ser feito em softwares como o Revit, o ArchiCAD, o Vectorworks, o AECOSim Architecture ou SketchupPro, por exemplo (WITICOVSKI, 2011). As quantidades são acuradas e referem-se às

informações dos materiais incorporados no modelo e que serão usados na construção da edificação.

Outra contribuição das ferramentas BIM para a construção de uma edificação é o uso de softwares específicos para orçamentação e planejamento de obras, como o VICO Office, o Primus Software e o Allplan, que conectam etapas importantes da gestão da edificação com os projetos em BIM. (BARISON, 2015; SAKAMORI, 2015)

Além desses, existem outros softwares BIM que merecem destaque para a construção de uma edificação. Esses softwares são voltados para a detecção de conflitos existentes entre os modelos de diferentes disciplinas, como as possiveis interferências entre arquitetura, estrutura e instalações. Softwares como Solibri Model Checker e Tekla BIM Sight contribuem para evitar que conflitos entre esses projetos passem despercebidos, e que as interferências sejam resolvidas antes de serem executadas na obra, reduzindo assim os gastos com retrabalhos, perdas de tempo, de material, de mão de obra e a possível geração de resíduos. (BARISON, 2015; MANZIONE, 2013)

No caso da manutenção e operação de uma edificação, os softwares YouBIM, Allplan Allfa e o Planon, permitem o controle pós-obra do empreendimento, através de cadastro prévio de equipamentos e demais itens desejados, e da realização do controle de datas importantes e períodos determinados de manutenção ou troca. Permite também o controle das quantidades de cada equipamento ou item, e outras funcionalidades que são integradas aos modelos BIM (YOUBIM, (20--?)).

\subsection{Viabilidade Econômica com uso de BIM}

As ferramentas BIM para estudo de viabilidade visam à estimativa inicial de custos e ao planejamento inicial de empreendimentos que estão na fase de concepção ou de estudo de viabilidade. Como resultados, as ferramentas auxiliam na tomada de decisão nas etapas iniciais de projeto, trazendo a estimativa preliminar de custos para execução da obra e a possibilidade de estudos de massa para decisão da forma arquitetônica. (BARISON, 2015)

Uma pesquisa realizada na Universidade de Brasília abordou formas de lançamento da arquitetura pensando no custo de uma edificação e utilizou o software DProfiler para obter os resultados. Após o desenvolvimento dos modelos propostos na pesquisa, comprovou-se que é possível determinar uma forma arquitetônica aliada a um estudo preliminar de custos, que neste caso foi possível com o auxílio do software DProfiler (GARCIA, 2014).

Garcia (2014, p.184), descreve ainda que "fica ressaltada também a utilidade de se pensar no custo antes de partir 
para os devaneios da forma quando se pensa em sustentabilidade", mostrando que a sustentabilidade em seu pilar econômico é importante para a tomada de decisão formal assertiva e pela busca da construção sustentável. A figura 5 mostra o desenvolvimento da forma arquitetônica da pesquisa deste autor, que iniciou com um formato de prisma retangular, cujo custo preliminar aproximado estava orçado em $\mathrm{R} \$ 105.000,00$ e chegou a um formato que contém intersecções e ajustes nos volumes da forma arquitetônica, com um custo preliminar de R\$ 208.510,65.

\subsection{Custo de Construção usando ferramen- ta BIM}

Figura 5 - Processo de modificação da forma arquitetônica no DProfiler para obtenção do custo preliminar.

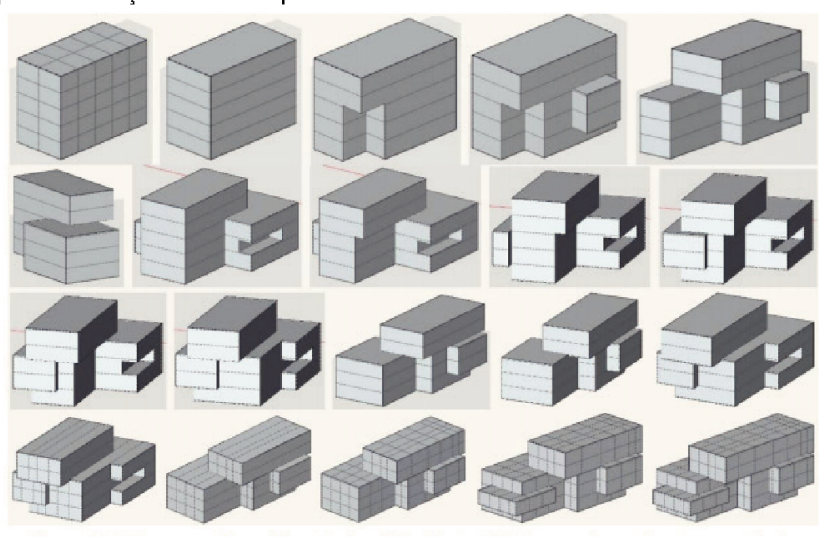

Fonte: Garcia, 2014

Em relação aos custos de construção, as ferramentas BIM contribuem desde a extração de quantidades até a execução do orçamento executivo. Os softwares BIM 3D, usados para modelagem dos projetos, a exemplo do ArchiCAD, AECOSim, Vectorworks e Revit, permitem a extração automática e praticamente imediata de quantitativos diretamente do modelo 3D. (BARISON, 2015; SAKAMORI, 2015)
O Revit é o software BIM 3D mais conhecido mundialmente. Pode ter interfaces para trabalhar com projetos de arquitetura (3D - Revit Architecture), estruturas (3D - Revit Structure) e instalações (3D - Revit MEP), além de permitir a extração das quantidades diretamente do modelo, conforme figura 6. (FERNANDES, 2014; MATTANA; LIBRELOTTO, 2016)

É importante considerar aqui duas questões a respeito da extração de quantidade a partir dos modelos 3D: a primeira é que o nível de detalhamento do modelo será essencial para a extração precisa e confiável das quantidades necessárias para um orçamento, e a segunda é que a qualidade do modelo também é de extrema importância para a obtenção dos valores das quantidades. (MANZIONE, 2013; MELHADO, S.; PINTO, 2015)

Por isso, antes de extrair as quantidades de um modelo 3D, é essencial utilizar softwares BIM para checagem de conflitos e incompatibilidades entre os modelos de diferentes disciplinas. Estas ferramentas, a exemplo do Solibri Model Checker, do Tekla BIM Sight e do Navisworks, possibilitam a modelagem com mais qualidade e mais próxima da realidade, e consequentemente, a extração de quantidades dos modelos mais acuradas. (MANZIONE, 2013)

O Tekla BIM Sight (figura 7) é um visualizador gratuito desenvolvido pela empresa Trimble e foi utilizado para checagem de conflitos do modelo estudado por Mattana e Librelotto (2016). É fácil de usar e permite a compatibilização e checagem da geometria do modelo BIM, para identificação de conflitos entre diferentes disciplinas de projetos.

Em relação ao orçamento com uso de softwares BIM, denominado de BIM 5D, existem outras ferramentas que são específicas para este uso, tais como o VICO Office, o Primus e o Allplan BCM. Através destas ferramentas é possível desenvolver um orçamento executivo integrado com um modelo desenvolvido em ferramentas BIM

Figura 6 - Modelagem 3D no Revit Architecture Student 2015 e quantidades extraídas do modelo.
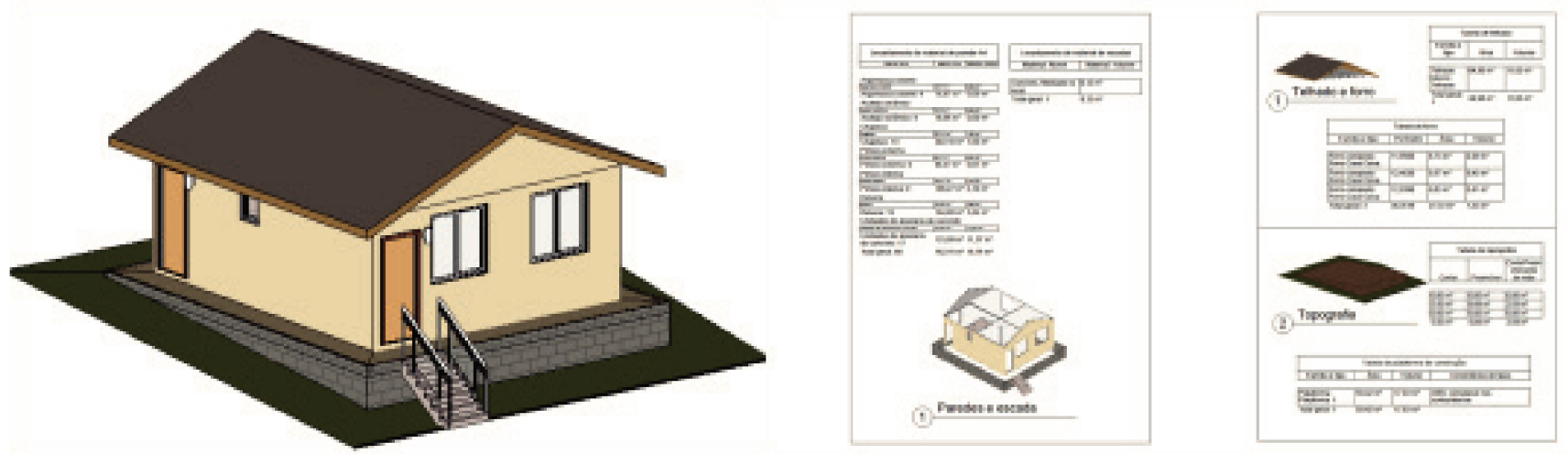

Fonte: Autoras 
3D. O modelo desenvolvido para o estudo de Mattana e Librelotto (2016) foi utilizado para experimentação da ferramenta VICO Office, conforme Figura 8.

Figura 7 - Interface do Tekla BIM Sight - checagem de conflitos do modelo 3D

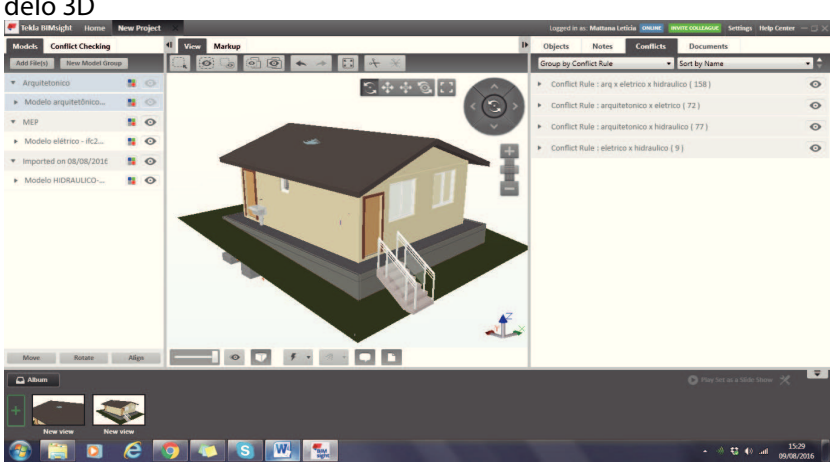

Fonte: Autoras

O Vico Office trabalha com BIM 4D e 5D, ou seja, com a gestão da construção nos parâmetros de planejamento e orçamento de obras. Com o Vico é possível estimar os custos, levantar quantidades do projeto e utilizar a linha de balanço para planejamento. Esta ferramenta é destinada à gestão de construção. (FERNANDES, 2014).

Figura 8 - Interface do VICO Office para BIM 5D

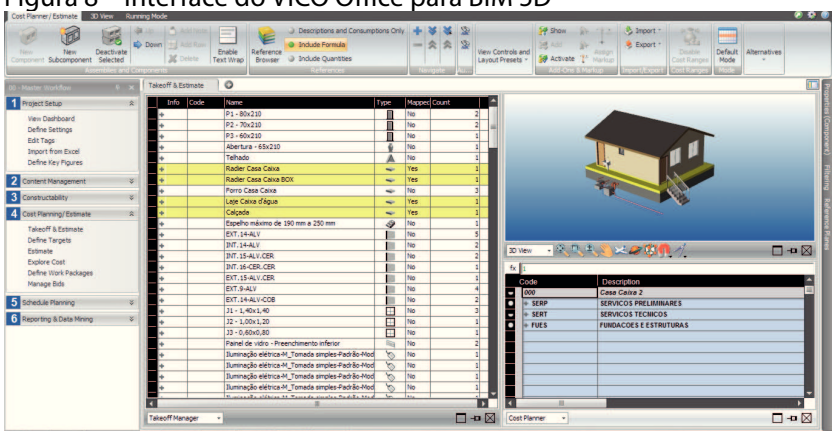

Fonte: Autoras

\subsection{Custo de Manutenção e Operação com ferramentas BIM}

As ferramentas BIM podem ser utilizadas também para a gestão da operação e manutenção de um empreendimento. Softwares como o YouBIM (figura 9) estão sendo usados com esta finalidade. Esta ferramenta oferece um banco de dados integrado que pode ser acessado na nuvem e contêm diversas informações sobre o edifício destinadas à manutenção predial, inclusive informações sobre manutenção rotineira de equipamentos. (YOUBIM, (20--?))

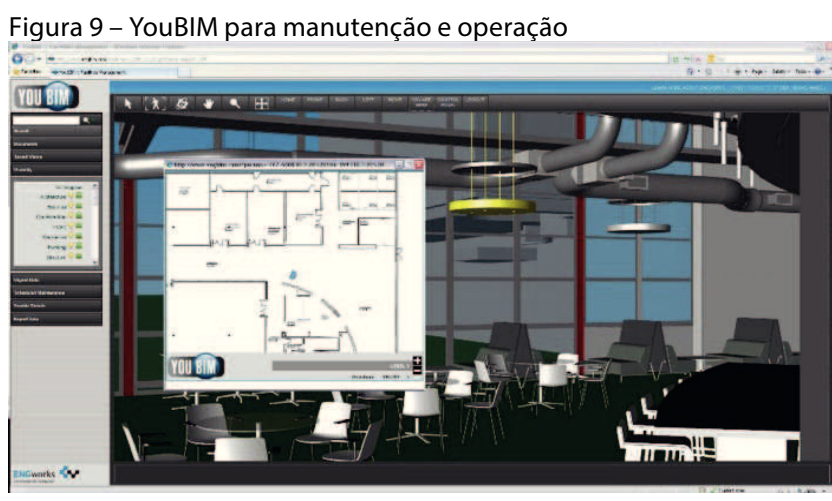

Fonte: YouBIM ((20--?))

\section{CONCLUSÕES}

Os resultados deste artigo demonstram que as ferramentas BIM possibilitam planejar e simular a sustentabilidade econômica de uma edificação de forma parcial, tomando como referência os parâmetros definidos pela Carvalho (2009) na metodologia MASP-HIS e que definem a sustentabilidade econômica.

A principal contribuição do BIM está na obtenção de informações para a viabilidade econômica e para os custos de construção/manutenção/operação de uma edificação.

Já para o fortalecimento da economia local e para os critérios econômicos para empresa de projeto, a tecnologia BIM não apresenta contribuição direta, ou seja, até o momento não foram localizadas ferramentas que auxiliem na obtenção destas informações específicas, cabendo as empresas de projetos optar por outras estratégias que não remetam ao uso de tecnologia BIM.

Outra importante consideração é que para que as ferramentas BIM tragam resultados precisos e assertivos sobre o custo de uma edificação (seja ele custo de construção, de manutenção, de operação ou a estimativa de custo para a viabilidade econômica de um empreendimento) a qualidade do modelo BIM deverá ser avaliada.

Um modelo que contém erros ou falta de padronização de modelagem poderá acarretar inconsistências nos resultados das quantidades extraídas do projeto, o que gera desvios nos custos finais. Por isso, é importante realizar a compatibilização entre os modelos antes de desenvolver o orçamento ou as estimativas com base no modelo BIM.

Outra importante análise refere-se à uma estrutura (hierarquia) de modelagem das famílias que deve possuir compatibilidade com a EDP (Estrutura de Decomposição de Projetos) utilizada como base para levantamento das quantidades para elaboração do orçamento.

De modo geral, a tecnologia pode trazer importantes contribuições ao reduzir o tempo de obtenção da informação e a complexidade de extração dos dados. 


\section{REFERÊNCIAS}

ABNT. Edificações habitacionais - Desempenho. TÉCNICAS, A. B. D. N. Brasília 2013.

AIA, D. E. Building Information Modeling Protocol Exhibit: The American Institute of Architects: 9p. p. 2008.

ALSAYYAR, B.; JRADE, A. Integrating Building Information Modeling (BIM) with sustainable universal design strategies to evaluate the costs and benefits of building projects. 5th International/11th Construction Specialty Conference. Vancouver - British Columbia: 10 p. 2015.

AZEVEDO, O. J. M. D. Metodologia BIM - Building Information Modeling na Direcção Técnica de Obras. 2009. 114p. (Mestrado). Engenharia Civil, Reabilitação, Sustentabilidade e Materiais de Construção, Universidade do Minho

BARISON, M. B. Introdução de Modelagem da Infromação da Construção (BIM) no currículo - uma contribuição para a formação do projetista. 2015. Universidade de São Paulo, São Paulo.

BRUNDTLAND, G. H. et al. Nosso Futuro Comum Relatório de Brundtland. Rio de Janeiro: FGV, 1987.

CAPES. Banco de Teses e Dissertações da CAPES. 2017. Disponível em: < http://bancodeteses.capes.gov.br/banco-teses/\#!/ >.

CARVALHO, M. T. M. Metodologia para avaliação da sustentabilidade de habitações de interesse social com foco no projeto. 2009. 241p. (Doutorado). Departamento de engenharia civil, Unversidade de Brasília, Brasília.

EASTMAN, C. et al. Manual de BIM: Um guia de modelagem a informação da construção para arquitetos, engenheiros, gerentes, construtores e incorporadores. Porto Alegre: Bookman, 2014. 483 ISBN 978-85-8260-117-4.

FERNANDES, C. A. P. Interoperacionalidade em sistemas de informação. 2014. 130p. (Mestrado). Universidade do Minho, Azurém, Portugal.
GARCIA, D. D. S. ARQUITETURA PERFORMATIVA: A UTILIZAÇÃO DO DPROFILER PARA ELABORAÇÃO DA FORMA ARQUITETÔNICA. . 2014. 240p. (Mestrado). Arquitetura e Urbanismo, Universidade de Brasília, Brasília.

IBGE, I. B. D. G. E. E.-. Indicadores de desenvolvimento sustentável. GEOGRAFIA, C. D. R. N. E. E. A. E. C. D. Rio de Janeiro: 352 p. 2015.

JOHN, V. M. Desenvolvimento sustentável e a construção de habitacional. . REUNIÃOANUAL DA SOCIEDADE BRASILEIRA PARA O PROGRESSO DA CIÊNCIA 58;

SIMPÓSIO ANTAC: O DESENVOLVIMENTO SUSTENTÁVEL E A CONSTRUÇÃO HABITACIONAL, 2006.

KASSEM, M.; AMORIM, S. R. L. D. BIM - BUILDING INFORMATION MODELING NO BRASIL E NA UNIÃO EUROPÉIA. Brasília. 2015

LIBRELOTTO, L. MODELO PARA AVALIAÇÃO DA SUSTENTABILIDADE NA CONSTRUÇÃO CIVIL NAS DIMENSÕES ECONÔMICA, SOCIAL E AMBIENTAL (ESA): APLICAÇÃO NO SETOR DE EDIFICAÇÕES. 2005. 371 (Doutorado). Universidade Federal de Santa Catarina, Florianópolis.

LIBRELOTTO, L.; SANON, S.; FERROLI, P. C. M. Avaliação da Sustentabilidade do Edifício na Escala Urbana: Modelo ESA Edificações. ENSUS 2017. Florianópolis 2017.

MANZIONE, L. Proposição de uma estrutura conceitual de gestão do processo de projeto colaborativo com o uso do BIM. . 2013. 325 (Doutorado). Escola Politécnica, USP, São Paulo.

MARCOS, M. H. C. Método de obtenção de dados de impactos ambientais, durante o processo de desenvolvimento do projeto, através do uso de ferramenta BIM. 2015. 145 (Doutorado). Faculdade de Arquitetura e Urbanismo, Universidade de São Paulo, São Paulo.

MATTANA, L.; LIBRELOTTO, L. I. The use of BIM technology in class: an experience at Federal University of Santa Catarina. BIC 2016. São Paulo 2016.

MELHADO, S.; PINTO, A. C. Benefícios e desafios da utilização do BIM para extração de quantitativos. SIBRAGEC - ELAGEC 2015. São Carlos/SP: 511-518 p. 2015. 
MELHADO, S. B.; UECHI, M. E.; DE PAULA, N. Novas demandas para as empresas de projeto de edifícios. Ambiente Construído, v. 13, p. 137-159, jul./set. 20132013.

NBIMS. National BIM Standard - United States. Washington, 2016. Disponível em: < https://www.nationalbimstandard.org/faqs\#faq1 >. Acesso em: 07-19.

NOUNPROJECT. The Noun Project. 2017. Disponível em: < https://thenounproject.com/ >.

OLIVEIRA, E. D.; SCHEER, S.; TAVARES, S. F. AVALIAÇÃO DE IMPACTOS AMBIENTAIS PRÉ-OPERACIONAIS EM PROJETOS DE EDIFICAÇÕES E A MODELAGEM DA INFORMAÇÃO DA CONSTRUÇÃO. TIC2015, 2015.

ROZENFELD, $H$. et al. Gestão de desenvolvimento de produtos: uma referência para a melhoria do processo. . São Paulo: Saraiva, 2006. 542 ISBN 8502054465.

SACHS, I. Estratégias de transição para o século XXI. São Paulo: Nóbel, 1993.

SAKAMORI, M. M. MODELAGEM 5D (BIM) CONTROLE DE CUSTOS E VALOR AGREGADO PARA EMPREENDIMENTOS DE CONSTRUÇÃO CIVIL. 2015. 180 (Dissertação de mestrado). PROGRAMA DE PÓSGRADUAÇÃO EM ENGENHARIA DE CONSTRUÇÃOCIVIL PPGECC, UNIVERSIDADE FEDERAL DO PARANÁ, CURITIBA.

SANTOS, A. D. P. L.; ANTUNES, C. E.; BALBINOT, G. B. Levantamento de quantitativos de obras: comparação entre o método tradicional e experimentos em tecnologia BIM. Iberoamericn Journal of Industrial Engineering, v. 6, n. 12, p. 134-155, 2014.

VIANA, K. R. C. Estudo do sistema construtivo em alvenaria estrutural na cidade do Rio de Janeiro face aos conceitos de construção sustentável. 2013. 131 (Mestrado). Departamento de Engenharia Civil., UFRJ, Rio de Janeiro.

WITICOVSKI, L. Levantamento de quantitativos em projeto: uma análise comparativa do fluxo de informações entre as representações em 2D e o modelo de informações da construção (BIM). 2011. 200p. (Dissertação de Mestrado). Programa de Pós-Graduação em Construção Civil, Universidade Federal do Paraná, Curitiba.

WONG, K.-D.; FAN, Q. Building information modelling (BIM) for sustainable building design. Facilities, v. 31, n. 3/4, p. 138-157, 2013.

YOUBIM. BIM para manutenção predial - Solução baseada na nuvem. (20--?). Disponível em: < http://www. youbim.com/br/features.html >. Acesso em: 15/11/2016. 